\title{
Das neue Weiterbildungsprogramm für Allgemeine Innere Medizin: Stärkung der Hausarztmedizin
}

\author{
Was lange währt, wird endlich gut. Die Plenarversammlung SIWF hat am 26. Novem- \\ ber 2009 das revidierte Weiterbildungsprogramm für Allgemeine Innere Medizin \\ verabschiedet. Dieses vollständig neu aufgebaute Weiterbildungsprogramm ist ge- \\ zielt auf den Bedarf der Bevölkerung ausgerichtet: breite Betreuung und Behand- \\ lung in der Ambulanz und im Krankenhaus.
}

Max Giger

Präsident SIWF

\begin{abstract}
Das Wichtigste in Kürze
Das Weiterbildungsprogramm umfasst drei Jahre Basisund zwei Jahre Aufbauweiterbildung. Die auf die Kompetenzen der künftigen Allgemeininternisten ausgerichtete modulare fünfjährige Weiterbildung schliesst nahtlos an die Ausbildungsziele des Medizinstudiums an und ermöglicht zwei Ausrichtungen, nämlich die Berufsausübung in der hausärztlichen Praxis (Curriculum Praxisinternist/Hausarzt) oder im Krankenhaus (Curriculum Spitalinternist). Die expliziten Lernziele strukturieren die Weiterbildung. Während der gesamten Weiterbildung soll ein formatives Assessment mit Feedback erfolgen.
\end{abstract}

\section{0-jährige Vorgeschichte}

Seit über zehn Jahren beschäftigen sich Fachgesellschaften und Weiterbildungsgremien mit der Gestaltung eines Weiterbildungsprogramms, das den Absolventen erlaubt, allgemeininternistische Patientinnen und Patienten im Krankenhaus und in der hausärztlichen Praxis zu betreuen. Die Überführung der inter-

\section{Es kommt zu einer inhaltlichen Neuausrichtung des Fachgebietes Innere Medizin. \\ Faktisch wird der Allgemeininternist geschaffen}

Korrespondenz:

Dr. med. Max Giger FMH/SIWF

Elfenstrasse 18

CH-3000 Bern 15

max.giger@hin.ch nistischen Schwerpunkte in Facharzttitel, die Bedürfnisse der grossen Krankenhäuser und die Bildung von fachübergreifenden Departementen führten zu einer inhaltlichen Neuausrichtung des Fachgebietes Innere Medizin. Es wurde faktisch der Allgemeininternist (internistischer «Housestaff») geschaffen. Diesem Umstand fiel der Vorschlag zur Schaffung eines hochspezialisierten Spitalinternisten [1] zum Opfer. In der Ambulanz betreuten Fachärzte für Innere Medizin und
Allgemeinmedizin mehr oder weniger identische Patienten. Die Weiterbildungsprogramme waren inhaltlich grossteils deckungsgleich. Die Arbeitsgruppe Ausund Weiterbildung des Kollegiums für Hausarztmedizin empfahl deshalb Ende 2007 die Schaffung eines einzigen Weiterbildungscurriculums. Aus universitären Kreisen [2] wurde eine Zusammenlegung der zwei Facharzttitel und Schaffung eines kompetenzbasierten modularen Weiterbildungsprogramms gefordert. Auf die Stellungnahme der Internisten [3] folgten unzählige Arbeitssitzungen und Gespräche bis zur Verabschiedung des modernen Weiterbildungsprogramms für Allgemeine Innere Medizin durch das SIWF am 26. November 2009. Dieses soll am 1. Juli 2010 in Kraft treten.

\section{Aufbau und Dauer der Weiterbildung}

Die Weiterbildung umfasst drei Jahre Basisweiterbildung und zwei Jahre Aufbauweiterbildung. Die dreijährige Basisweiterbildung muss mindestens zwei Jahre stationäre Allgemeine Innere Medizin und mindestens ein halbes Jahr ambulante Allgemeine Innere Medizin, vorzugsweise Praxisassistenz, beinhalten. Die Aufbauweiterbildung besteht aus mindestens zwei individuell wählbaren Modulen zur Komplettierung der Weiterbildung zum Spitalinternisten oder Praxisinternisten bzw. Hausarzt. Das Curriculum ist so aufgebaut, dass vorzugsweise zuerst die Basisweiterbildung absolviert wird. So kann danach die Aufbauweiterbildung auf die spätere Berufstätigkeit ausgerichtet werden. Die sogenannten «Hausarztprogramme», die in der Westschweiz, am Kantonsspital St.Gallen und neu am UniversitätsSpital Zürich angeboten werden, sind mit dem neuen Weiterbildungsprogramm Allgemeine Innere Medizin vollumfänglich kompatibel. Bei entsprechender Planung kann die Weiterbildung innert fünf Jahren abgeschlossen werden. Es wird ein einziger Facharzttitel für Allgemeine Innere Medizin verliehen. 


\section{Ziele der Weiterbildung}

Die Weiterbildung soll die notwendigen Kompetenzen vermitteln, eigenverantwortlich die medizinische Grundversorgung und fachärztliche Betreuung der Patienten sicherzustellen. Des Weiteren soll die notwendigen Kompetenzen vermittelt werden, um unter richtiger Einschätzung des Nutzen-Risiko- und Kosten-Nutzen-Verhältnisses die erforderlichen diagnostischen, präventiven und therapeutischen Massnahmen unter Einbezug von psychischen, sozialen und kulturellen Gesichtspunkten durchzuführen oder anzuordnen.

Die Lernziele werden detailliert aufgeführt. Die allgemeinen Lernziele sind nach den in CanMeds [4] definierten sieben Rollen des Arztes in der Weiterbildungsordnung (WBO) festgehalten. Die fachspezifischen allgemeininternistischen Lernziele und diejeni- ment während der gesamten Weiterbildung. Die Weiterzubildenden erhalten so eine Rückmeldung über den Stand der Weiterbildung zusammen mit einer Empfehlung für die nächsten Lernziele. Die Einführung von AbA soll nach Schulung der Weiterbildner schrittweise erfolgen.

\section{Einführung dieses ersten kompetenzbasierten Weiterbildungsprogramms}

Das neue Weiterbildungsprogramm soll per 1. Juli 2010 in Kraft treten. Bis zu jenem Zeitpunkt müssen noch das Logbuch, das Konzept für AbA und die Einteilung der Weiterbildungsstätten erstellt bzw. durchgeführt werden. Die Verantwortlichen für die Weiterbildung an allen Weiterbildungsstätten sollen über die Einführung dieses modernen Weiterbildungsprogramms de-

\section{Die Einführung dieses modernen kompetenzbasierten Weiter- bildungsprogramms für Allgemeine Innere Medizin möge weitere Fachgesellschaften zur Nachahmung animieren}

gen der nichtinternistischen Fächer basieren auf dem Lernzielkatalog der Schweizerischen Medizinischen Interfakultätskommission (SMIFK) [5]. Im Rahmen der Basisweiterbildung werden Basiskompetenzen vermittelt u. a. in der Behandlung von Patienten bzw. Patientinnen mit häufigen dermatologischen, gynäkologischen und otorhinolaryngologischen Problemen. Ebenfalls eingeschlossen sind «kleine Wundversorgungen».

Der Lernzielkatalog strukturiert die Weiterbildung und erlaubt den Weiterzubildenden ein zielstrebiges Erwerben der notwendigen Kompetenzen. Für die nichtinternistischen Fächer (u.a. Chirurgie, Psychiatrie, Kinder- und Jugendmedizin, Gynäkologie und Geburtshilfe) umfasst der Katalog die während einer sechsmonatigen Weiterbildungsphase mindestens zu vermittelnden Lerninhalte. Die Leiter von Weiterbildungsstätten, namentlich in den nichtinternistischen Fächern, sollen die Weiterbildung auf die genannten Ziele ausrichten und dies in ihren Weiterbildungskonzepten festhalten. Dadurch wird neu die Weiterbildung in Hausarztmedizin effizient strukturiert.

\section{Logbuch und Arbeitsplatz-basiertes Assessment}

Die Weiterzubildenden werden angehalten, ein Logbuch zu führen, in dem sie die vermittelten bzw. erworbenen Lerninhalte festhalten. So können sie sich einerseits über den Grad der Erfüllung der obligatorischen Weiterbildung orientieren und andererseits eigenverantwortlich ihr Weiterbildungscurriculum gestalten. In diesem Logbuch soll auch das arbeitsplatzbasierte Assessment mit Feedback (AbA) [6, 7] festgehalten werden. AbA ermöglicht ein formatives Assess- tailliert orientiert werden. Nur so können die obgenannten Ziele, namentlich die Effizienzsteigerung in der Weiterbildung im Allgemeinen und die Stärkung der Hausarztmedizin im Speziellen, erreicht werden. Die erste schriftliche summative Prüfung (Wahlantwortfragen) wird 2012 stattfinden. Diese soll durch eine gemischte Prüfungskommission mit Vertretern aus den Gesellschaften für Innere Medizin und Allgemeinmedizin in Zusammenarbeit mit dem Institut für Medizinische Lehre (IML) der Universität Bern erarbeitet werden.

Die Einführung dieses modernen kompetenzbasierten Weiterbildungsprogramms für Allgemeine Innere Medizin möge weitere Fachgesellschaften zur Nachahmung animieren.

\section{Literatur:}

1 de Torrenté A. Achtung: Verwechslungsgefahr! Schweiz Med Forum 2002(47):1113-4.

2 Perrier A. et al. Plädoyer für eine gemeinsame Fachgesellschaft für Allgemeinmedizin und Innere Medizin in der Schweiz. Schweiz Ärztezeitung 2008;89(14):516-22. 566-9.

3 Briner A. Stellungnahme der SGIM. Schweiz Ärztezeitung 2008;89(14):569-71.

4 http://rcpsc.medical.org/canmeds/CanMEDS2005/ index.php

5 Swiss Catalogue of Learning Objectives for Undergraduate Medical Training - June 2008 (www.smifk.ch).

6 Berendonk C, Beyeler C, Westkämper R, Giger M. Strukturiertes Feedback in der ärztlichen Weiterbildung: Mini-CEX und DOPS. Schweiz Ärztezeitung 2008;89(32):1337-40.

7 PMETB.Workplace Based Assessment (WPBA): A Guide for Implementation. www.pmetb.org.uk/ index.php?id=wpba (accessed 2009-11-22). 